\title{
Variations de la composition de la végétation herbacée des plantations de Acacia senegal (L.) Willd de la zone de Dahra (Ferlo)
}

\author{
Aly DIALLO ${ }^{1}$, Madiara NGOM FAYE ${ }^{1}$, Ousmane NDIAYE ${ }^{1}$ et Aliou GUISSE ${ }^{1,2^{*}}$ \\ ${ }^{1}$ Département de Biologie Végétale, Faculté des Sciences et Techniques, Université Cheikh Anta Diop, \\ B.P. 5005. Dakar-Fann, Sénégal. \\ ${ }^{2}$ Observatoire Homme Milieu Tessekéré (Ferlo) \\ *Auteur correspondant, E-mail : aliou.guisse@ucad.edu.sn; alguisse@orange.sn; Tel : 221776389669.
}

\section{RESUME}

Cette étude se propose d'établir la variabilité floristique de la végétation herbacée dans les plantations de Acacia senegal (L.) Willd de la zone de Dahra à la limite sud du Ferlo sableux. Des relevés phytosociologiques ont été effectués et traités par les méthodes d'analyse multivariée (AFC). Les résultats qui ressortent de l'analyse factorielle de correspondance (AFC), mettent en évidence une assez forte variabilité de composition floristique riche de 19 familles représentées par 42 genres et 62 espèces. Cette variabilité est en relation avec l'hétérogénéité de la végétation et les fluctuations des facteurs écologiques. Cela a permis de caractériser sur le plan spatial quatre groupements végétaux fortement tributaires des facteurs topographiques, édaphiques et de la disponibilité de l'eau. Les facteurs anthropiques, à travers la forte pression de pâture dans les plantations qui ne bénéficient pas d'une protection intégrale, modifient considérablement la composition floristique de la strate herbacée. Dans les plantations moins soumises à l'action du bétail, la strate herbacée forme un tapis continu et est source de fourrage durant les périodes difficiles de l'année où les zones de pâturages sont quasi inexistantes.

(C) 2011 International Formulae Group. All rights reserved.

Mots clés: Strate herbacée, variabilité floristique, phytosociologie, Acacia senegal, AFC, Ferlo.

\section{INTRODUCTION}

Depuis plus de dix ans la sècheresse persiste dans la zone du Sahel, en particulier dans le Ferlo; les possibilités de reconstitution du milieu naturel semblent de plus en plus réduites, du fait que les causes naturelles et les causes anthropiques se conjuguent pour maintenir et étendre les dégradations du couvert végétal. Les changements notables induits dans les écosystèmes entrainent une précarisation de plus en plus accentuée des conditions de vie des populations rurales qui sont les plus affectées par les effets de cette désertification, du fait que la végétation spontanée constitue la base de l'alimentation des troupeaux et que la production vivrière repose sur la culture pluviale. 
La végétation et les sols ont fait l'objet de nombreuses recherches, notamment sur l'inventaire floristique, la typologie et la cartographie (Trochain, 1940; Raynal, 1964; Penning de Vries et Djiteye, 1982; Barral et al., 1983; Chevallier et al., 1985; Grouzis, 1988).

Bien que la variabilité caractéristique du milieu sahélien soit peu connue, des recherches comme celles de Cornet (1981) et Barral et al. (1983) ont mis en évidence des variations de la structure spécifique. Les fluctuations spatio-temporelles des cycles de production (Grouzis, 1988) ont été mises en relation avec les conditions édaphoclimatiques. L'étude des mécanismes responsables de la mise en place du peuplement herbacé (Grouzis et al., 1986; Carrière, 1989), ou des déterminismes hydriques (Cornet, 1981) et trophiques (De Vries et Djiteye, 1982) de la production végétale a permis de proposer des modèles de production des pâturages (Hiernaux, 1984).

La phénologie des ligneux a été reliée aux variations des conditions écologiques (Poupon, 1980) et la productivité d'un certain nombre d'espèces ligneuses a été évaluée (Poupon, 1980 ; Toutain et al., 1983).

Une meilleure gestion des réserves sylvo-pastorales passerait par la restauration et (ou) la réhabilitation des écosystèmes dégradés (Akpo et Grouzis, 1996). Celle-ci doit reposer sur la connaissance de l'état actuel de ces ressources afin de concilier au mieux les nécessités de la production herbacée (cultures, espèces pastorales) et la stabilité du milieu apportée par les ligneux du fait qu'ils soient moins sensibles aux perturbations de l'environnement (Menaut, 1983).

C'est dans ce cadre que nous envisageons d'apporter notre contribution à l'étude de la flore et de la végétation du Ferlo, afin de fournir des informations qui pourraient aider à une meilleure connaissance de la végétation et une meilleure compréhension du rôle de certains facteurs écologiques, en particulier les facteurs topographiques, édaphiques et anthropiques dans la différenciation des groupements végétaux. L'objectif de l'étude est donc d'étudier la variation floristique de la végétation herbacée dans les plantations d'Acacia senegal (L.) Willd de la zone de Dahra, et de caractériser les différents groupements végétaux.

\section{MATERIEL ET METHODES \\ Présentation de la zone d'étude}

L'étude a été menée dans la partie sud du Ferlo sableux, à Dahra $\left(15^{\circ} 20^{\prime} \mathrm{N}\right.$ et $15^{\circ}$ $28^{\prime} \mathrm{O}$ ), localité située à environ $270 \mathrm{~km}$ au Nord-Est de Dakar et à $50 \mathrm{~km}$ à l'Ouest de Linguère. Dahra est une des communes du département de Linguère. $\mathrm{Au}$ cours des missions de prospection, quatre sites ont été retenus. Il s'agit des plantations: Isra 1999 qui se situe dans le centre de recherche zootechnique de Dahra (CRZ) à une dizaine de kilomètres à l'est de la ville, Ndodj 2001 à environ $24 \mathrm{~km}$ au nord-est, Boulal 2004 à 20 km à l'ouest et Déali 2005 à environ 45 km au Sud-Ouest (Carte 1).

L'effet de la topographie sur la variation de la composition de la strate herbacée sera étudié en comparant les positions de dune et de bas fond dans les sites Isra et Déali caractérisés par une alternance de dunes, de dépressions et de zone de replat. Si Ndodj se caractérise par un relief plat, Boulal par contre est marqué par de petites dépressions interdunaires. L'effet de l'humidité sera évalué par comparaison de certains sites du Nord (Ndodj par exemple) avec ceux du Sud (Isra par exemple). Pour étudier l'effet de ce facteur, nous avons retenu Ndodj (pluviométrie faible) et Isra (pluviométrie élevée). Entre 2001 et 2005, la pluviosité moyenne est de $202 \mathrm{~mm}$ à Ndodj, par contre à la station Isra elle est de $293 \mathrm{~mm}$. Par ailleurs l'effet de la protection sera évalué dans les sites dont certains, malgré la mise en défens, ne bénéficient pas d'une protection intégrale (surveillance régulière), comme c'est le cas à Déali qui est une zone de parcours. Les plantations Isra et Boulal sont intégralement protégées tandis que Ndodj est partiellement protégée. 
Le climat est de type sahélien, la température moyenne annuelle est de $28,6{ }^{\circ} \mathrm{C}$ et les températures moyennes mensuelles minimale et maximale sont respectivement de $24,4{ }^{\circ} \mathrm{C}$ (janvier) et $32,3{ }^{\circ} \mathrm{C}$ (mai). Depuis 1970 , il y a une tendance à la hausse des températures de 0,1 à $1,8^{\circ} \mathrm{C}(\mathrm{CSE}, 2002)$. Le régime pluviométrique se situe entre les isohyètes 100 et $500 \mathrm{~mm}$. La pluviosité moyenne de la station de référence (Linguère) est de $282 \mathrm{~mm}$, avec un coefficient de variation de $37 \%$. Comme d'autres zones sahéliennes, Linguère se caractérise par un déficit pluviométrique particulièrement sévère depuis 1970 (CSE, 2002). En 2005, la pluviométrie a été de $235 \mathrm{~mm}$ dont $80 \%$ pour les mois de juillet et août. Les pluies s'étendent en effet de juin à octobre (Figure 1); et dans l'année, on distingue classiquement deux périodes: une période sèche de sept à neuf mois (octobre à mai) et une saison des pluies de 3 à 5 mois.

Le gradient pluviométrique crô̂t du nord (Ndodj) vers le Sud (Déali), avec une forte variabilité interannuelle (Figure 2).

Cette zone fait partie d'un ensemble dunaire fortement érodé, au relief très faible, où Valenza et Diallo (1972) distinguent sept formations pédologiques, treize groupements et quarante huit parcours botaniques, constitués de plusieurs faciès. Ces formations de dunes de sables se caractérisent par un ensemble de rides asymétriques séparées par des dépressions longitudinales à sol sablo-argileux grisâtre localement calcaire et à sol hydromorphe à engorgement temporaire (Michel, 1969). Quatre groupes de sols sont répertoriés: les sols ferrugineux tropicaux, les sols hydromorphes, les régosols et les lithosols sur cuirasse (CSE, 2002).

D'après les enquêtes menées par une équipe dirigée par Barral et al. (1983), la végétation du Ferlo aurait été constituée par une savane soudanienne fortement boisée où poussaient des herbes vivaces jusque dans les années 1970. La zone s'est sahélisée à cause des déficits pluviométriques successifs. En fin de saison des pluies, la végétation se présente sous la forme d'un tapis herbacé continu piqueté d'arbres et d'arbustes fréquemment épineux ne formant jamais de strate continue. De nombreuses espèces ligneuses telles que Terminalia avicennoides, Sterculia setigera, Sclerocarya birrea ou Combretum glutinosum n'apparaissent plus que sous forme d'individus morts sur pied ou se sont raréfiées; par contre d'autres espèces sont restées stables ou sont en extension, il s'agit de Balanites aegyptiaca, Acacia senegal, Boscia senegalensis. Beaucoup d'espèces herbacées à affinité soudanienne ont nettement régressé tandis que d'autres plus saharo-sahéliennes ont profité de la sécheresse pour s'étendre. Le pâturage et l'ébranchage pratiqués de manière excessive, ont en outre accentué les effets de la sécheresse

\section{Collecte des données}

Nous avons travaillé sur le sol et le tapis herbacé des différents sites d'étude afin de collecter des données édaphiques et floristiques qui sont soumises à des analyses multivariées.

\section{Données physicochimiques}

Les échantillons de sols ont été séchés dans des salles appropriées. Le sol est broyé puis tamisé à travers des mailles de dimensions variables. La terre fine obtenue est conservée dans des sachets en plastique sur lesquels les références des échantillons sont mentionnées. À partir de cette terre fine sont déterminés les paramètres suivants: $\mathrm{pH}$, bases échangeables, bilan ionique et granulométrie.

Toutes les analyses ont été effectuées au laboratoire GLA (Groupe de Laboratoire d'Analyses, Ministère des Mines de l'Industrie et des PME).

\section{Données floristiques}

L'inventaire de la flore a été effectué en 2009. Les échantillons des espèces ont été collectés, identifiés et actualisés dans l'herbier du département de biologie végétale de l'Université C.A. Diop de Dakar. Les taxons (genres et espèces) ont été déterminés d'après 


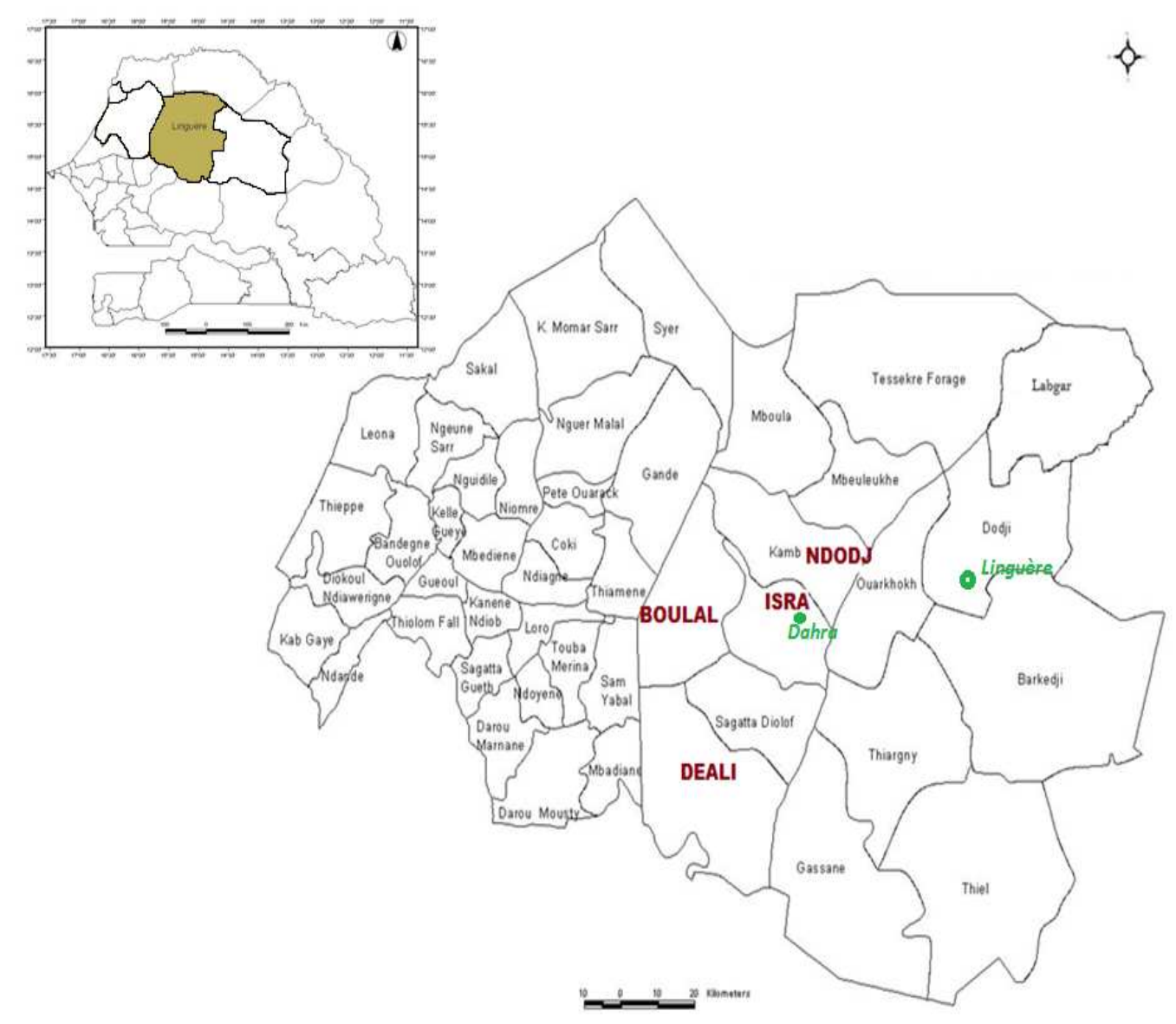

Carte 1 : Carte de situation de Linguère et des sites étudiés (PEPAM, 2007).

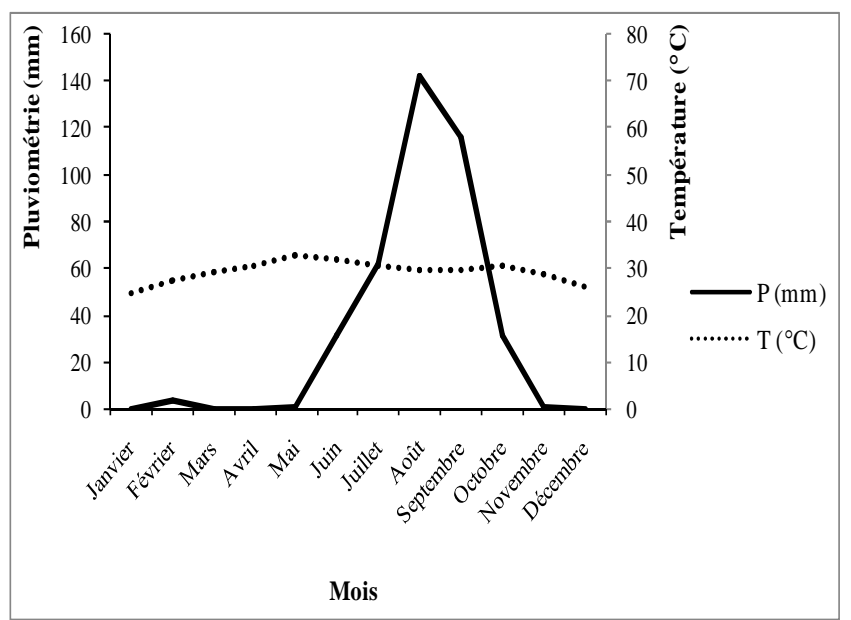

Figure 1 : Diagramme ombrothermique de Dahra de 1987 à 2005 (station de Linguère). 


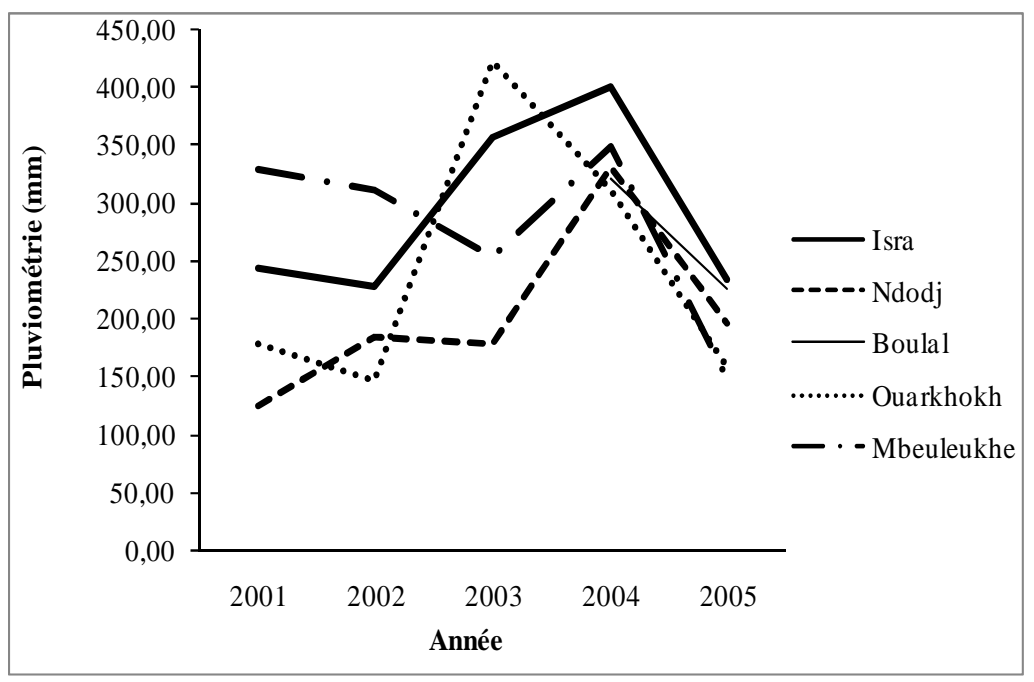

Figure 2 : Variabilité pluviométrique dans les différentes plantations de Acacia senegal de Dahra (2001 à 2005).

«les Flores du Sénégal» (Berhaut, 19711979), et actualisés d'après «l'Énumération des Plantes à Fleurs d'Afrique Tropicale » (Lebrun et Stork, 1991-1997).

La méthode d'investigation des groupements végétaux utilisée est basée sur le principe de la méthode sigmatiste des relevés phytosociologiques dont l'objectif est de déterminer les différents groupements végétaux du milieu d'étude (Braun-Blanquet, 1928 ; Guinochet, 1967, 1973). Elle repose sur le choix de zones floristiquement et topographiquement homogènes (Guinochet, 1967; Lacoste et Salanon, 1969) et dont la surface est au moins égale à l'aire minimale fixée entre 16 et $45 \mathrm{~m}^{2}$ pour la strate herbacée de différentes formations tropicales selon Poissonet et César (1972), Grouzis (1988) et Fournier (1991). Ainsi nous avons effectué 81 relevés phytosociologiques de $50 \mathrm{~m}^{2}$ à raison d'une vingtaine de relevés par plantation. Pour chaque relevé, nous notons les caractères non floristiques (situation géographique, géologie, pédologie, topographie) et les caractères floristiques concernant l'indice d'abondancedominance, le recouvrement, la sociabilité, la vitalité et la phénologie.

\section{Traitement des données}

Afin de dégager les similitudes écologiques et/ou floristiques éventuelles, les matrices espèces/relevés et groupements végétaux/paramètres physico-chimiques ont été soumises à une analyse factorielle de correspondance (AFC) (Guinochet, 1973; Dervin, 1990). Le traitement des données a été réalisé avec le logiciel XLSTAT.

\section{RESULTATS}

La flore inventoriée, dans ces quatre plantations, est riche de 62 espèces réparties dans 42 genres et 19 familles. Les genres les mieux représentés sont les genres Ipomoea (11,9\%), Eragrostis, Achyranthes, Cassia, Indigofera, Hibiscus et Spermacoce (7,1\%), les genres Aristida, Cucumus, Leptadenia et Momardica $(4,8 \%)$. Quant aux familles, nous avons dénombré pour les Poaceae 13 espèces soit $21 \%$, pour les Fabaceae 9 espèces soit $15 \%$, les Convolvulaceae 7 espèces soit $11 \%$, et les Cucurbitaceae 5 espèces soit $8 \%$ (Tableau 1). 
Variabilité floristique

L'analyse factorielle des correspondances a été appliquée à la matrice 81 relevés x 62 espèces végétales (Figure 3 ).

La variance, qui permet de quantifier la part d'information contenue par chaque axe, est portée par le Tableau 2.

$\mathrm{Ce}$ tableau montre que les quatre premiers axes expliquent environ $34 \%$ de la variance du nuage des points, dont $13 \%$ pour le premier axe; le second axe expliquant $8 \%$ de la variabilité totale.

La représentation des 81 relevés est fournie par la Figure 3.

L'agencement des relevés dans le plan factoriel principal montre une très nette séparation suivant l'axe $\mathrm{I}$, de deux groupes de relevés. Le premier groupe $\mathrm{G}_{1}$ est formé des relevés de Isra beaucoup plus humides (abscisses positives) et le second $\mathrm{G}_{2}$ des relevés de Déali et Boulal plus secs (abscisses négatives). Le facteur de variation semble donc l'humidité.

La distribution des relevés de Déali et Boulal le long de l'axe II permet de distinguer très nettement les relevés inféodés à Déali (abscisses positives) beaucoup plus exposé aux actions anthropiques de ceux caractéristiques de Boulal (abscisses négatives) qui bénéficie d'une certaine protection. Les relevés correspondant à Ndodj se situent en position intermédiaire. Ce deuxième axe représenterait donc le gradient de dégradation.

Les relevés de Isra sont associés à des espèces de zones humides (bas-fonds et bordure de cuvette) comme Basela alba L., Achyranthes argentea Lam. var. borbonica (Willd.), Brachiaria ramosa (L.) Stapf, Antigonon leptopus Hook. \& Arn., Mukia maderaspatana (L.) M.Roem., Hibiscus physaloides Guill. \& Perr., Cissus rufescens Guill. \& Perr., Cucumis melo var. agrestis Naudin, Achyranthes argentea Lam, Oxystelma bornouense R.Br., Ipomoea pestigridis L., Ipomoea asarifolia (Desr.) Roem. \& Schult., Indigofera astragalina DC. et Momordica balsamina L. Ces espèces forment le groupement à Achyranthes argentea Lam et à Brachiaria ramosa (L.) Stapf qui, de par leur contribution et coefficient d'abondance dominance, constituent les espèces les plus représentatives de ces relevés de bas-fonds et bordure de cuvette. Ce groupement est observé également dans les bas-fonds à Déali et Boulal mais sous une forme dégradée.

Quant aux relevés de Déali, ils sont associés à des espèces moins exigeantes en eau comme Mitracarpus villosus (Sw.) DC., Waltheria indica L., Spermacoce radiata (DC.) Hiern, Mollugo nudicaulis Lam., Tephrosia purpurea (L.) Pers., Chrozophora senegalensis (Lam.) A.Juss. ex Spreng., Ipomoea coptica (L.) Roth ex Roem. \& Schult., Eragrostis pilosa (L.) P.Beauv., Peristrophe paniculata (Forssk.) Brummitt, tandis que ceux de Boulal sont associés à Aristida adscensionis L., Schoenefeldia gracilis Kunth, Spermacoce ruelliae DC., Ipomoea kotschyana Hochst. ex Choisy et Panicum laetum Kunth. Les espèces rencontrées à Déali et Boulal sont caractéristiques des zones dégradées.

L'analyse partielle des relevés du groupe 2 (Déali, Ndodj et Boulal) permet de discriminer le long de l'axe I (Figure 4) les relevés de pentes et de bas de pente des relevés de dunes et zones de replat.

Sur les dunes et zones de replat se rencontrent les espèces comme Zornia glochidiata Rchb. ex DC., Ceratotheca sesamoides Endl., Striga hermonthica (Delile) Benth., Abildgaardia hispidula (Vahl) Lye, Aristida stipoides Lam., Sesamum indicum L., Indigofera pilosa Poir., Cassia obtusifolia L., Leptadenia hastata (Pers.) Decne., Aristida adscensionis L., Ipomoea kotschyana Hochst. ex Choisy, Panicum laetum Kunth, Schoenefeldia gracilis Kunth, Jacquemontia tamnifolia (L.) Griseb., Alysicarpus ovalifolius (Schumach. \& Thonn.) J.Léonard, Spermacoce ruelliae DC., Eragrostis tremula Hochst. ex Steud., Cenchrus biflorus Roxb. Les relevés de pentes et de bas de pente sont associés à des espèces comme Tephrosia purpurea (L.) Pers., Mitracarpus villosus 
(Sw.) DC., Waltheria indica L., Spermacoce radiata (DC.) Hiern, Mollugo nudicaulis Lam., Chrozophora senegalensis (Lam.) A.Juss. ex Spreng., Ipomoea coptica (L.) Roth ex Roem. \& Schult., Eragrostis pilosa (L.) P.Beauv., Peristrophe paniculata (Forssk.). Ces espèces sont rencontrées à Déali et forment le groupement à Tephrosia purpurea (L.) Pers. et Waltheria indica $\mathrm{L}$.

L'axe vertical II (Figure 4) distingue nettement deux groupes dans les relevés de dunes et de zones de replat. Le premier groupe est constitué dans les ordonnées positives, des relevés de replat tandis que le second, dans les ordonnées négatives représente l'ensemble des relevés de dunes. Les relevés de replat sont associés à Zornia glochidiata Rchb. ex DC., Ceratotheca sesamoides Endl., Striga hermonthica (Delile) Benth., Abildgaardia hispidula (Vahl) Lye, Aristida stipoides Lam., Sesamum indicum L. Indigofera pilosa Poir., Alysicarpus ovalifolius (Schumach. \& Thonn.) J.Léonard, Cenchrus biflorus Roxb. Ces espèces constituent le groupement à Zornia glochidiata Rchb. ex DC. Ce groupement est retrouvé à Ndodj et à Isra. Les relevés de dunes sont constitués par les espèces comme Cassia obtusifolia L., Leptadenia hastata (Pers.) Decne., Aristida adscensionis L., Ipomoea kotschyana Hochst. ex Choisy, Panicum laetum Kunth, Schoenefeldia gracilis Kunth, Jacquemontia tamnifolia (L.) Griseb., Alysicarpus ovalifolius (Schumach. \& Thonn.) J.Léonard, Spermacoce ruelliae DC., Eragrostis tremula Hochst. ex Steud.. Ces espèces forment le groupement à Aristida adscensionis L. et Schoenefeldia gracilis Kunth surtout à Boulal. A Déali, des espèces isolées sur butte ont été observées, il s'agit de Leptadenia pyrotechnica (Forssk.) Decne. et Cleome viscosa L. qui sont caractéristiques de cette zone.

\section{Caractéristiques physicochimiques des groupements}

Après identification des groupements, des échantillons de sols ont été prélevés dans les zones où ces groupements ont été rencontrés. Ces échantillons ont été par la suite analysés au laboratoire.

Le tableau de données portant en lignes les et en colonnes les mesures des paramètres physicochimiques correspondants (Tableau 3) a été soumis à une analyse factorielle de correspondance (Figure 5).

Les deux premiers axes définis par l'AFC expliquent $98,88 \%$ de la variabilité totale. L'axe horizontal $F_{1}$ permet de discriminer le groupement à Zornia glochidiata Rchb. ex DC. en abscisses positives et le groupement à Aristida adscensionis L. et Schoenefeldia gracilis Kunth en abscisses négatives. Le groupement à Zornia glochidiatia Rchb. ex DC. se caractérise par un sol sableux homogène légèrement alcalin, minéralisé à faible réserve de matière organique avec un rapport $\mathrm{Mg} / \mathrm{K}$ normal, et $\mathrm{Ca} / \mathrm{Mg}$ normal à optimum. Le groupement à Aristida adscensionis L. et Schoenefeldia gracilis Kunth est associé à un sol sableux hétérogène fortement alcalin, minéralisé à faible réserve de matière organique avec un rapport $\mathrm{Ca} / \mathrm{Mg}$ normal, $\mathrm{Mg} / \mathrm{K}$ normal à optimum mais le rapport $\mathrm{Ca}+$ $\mathrm{Mg} / \mathrm{K}$ est trop fort, ce qui atteste d'un déséquilibre entre les cations échangeables. L'axe $F_{2}$ oppose le groupement à Zornia glochidiatia au groupement à Tephrosia purpurea (L.) Pers. et Waltheria indica L. caractérisé par un sol sableux homogène fortement alcalin, minéralisé à faible réserve de matière organique dont le rapport $\mathrm{Ca}+$ $\mathrm{Mg} / \mathrm{K}$ est trop fort et les rapports $\mathrm{Ca} / \mathrm{Mg}$ et $\mathrm{Mg} / \mathrm{K}$ normaux.

Lorsqu'on s'intéresse au plan formé par les axes $\mathrm{F}_{1} \times \mathrm{F}_{3}$, on constate que l'axe $\mathrm{F}_{3}$ oppose le groupement à Tephrosia purpurea (L.) Pers. et Waltheria indica L. au groupement à Achyranthes argentea Lam et à Brachiaria ramosa (L.) Stapf caractérisé par un sol sableux hétérogène neutre à légèrement alcalin, minéralisé à faible réserve de matière organique; les rapports $\mathrm{Ca} / \mathrm{Mg}$ et $\mathrm{Ca}+\mathrm{Mg} / \mathrm{K}$ sont normaux à optimum, celui $\mathrm{Mg} / \mathrm{K}$ est normal. 
Tableau 1 : Liste des espèces herbacées, familles et codes correspondants.

\begin{tabular}{|c|c|c|}
\hline Familles & Noms scientifiques des espèces & Codes espèces \\
\hline Acanthaceae & Peristrophe paniculata (Forssk.) Brummitt & Ppa \\
\hline Molluginaceae & Mollugo nudicaulis Lam. & Mnu \\
\hline \multirow{3}{*}{ Amaranthaceae } & Achyranthes argentea Lam. & Aag \\
\hline & $\begin{array}{l}\text { Achyranthes argentea Lam. var borbonica Willd. ex } \\
\text { Schult. }\end{array}$ & Aba \\
\hline & Achyranthes aspera Lam. & Aas \\
\hline \multirow{3}{*}{ Apocynaceae } & Leptadenia hastata (Pers.) Decne. & Lha \\
\hline & Leptadenia pyrotechnica (Forsk.) Dec. & Lpy \\
\hline & Oxystelma bornouense R. Br. & Obr \\
\hline Asteraceae & Blainvillea gayana Cass. & Bga \\
\hline Basellaceae & Basella alba $\mathrm{L}$. & Bal \\
\hline \multirow{9}{*}{ Fabaceae } & Alysicarpus ovalifolius (Schumacher \& Thonning) & Aov \\
\hline & Cassia mimosoides $\mathrm{L}$. & $\mathrm{Cmi}$ \\
\hline & Cassia nigricans Vahl & Cni \\
\hline & Cassia obtusifolia $\mathrm{L}$. & $\mathrm{Cob}$ \\
\hline & Indigofera astragalina DC. & Ina \\
\hline & Indigofera pilosa Poir. & Inp \\
\hline & Indigofera senegalensis Lam. & Ise \\
\hline & Tephrosia purpurea (L.) Pers. (L.) Pers. & Tpu \\
\hline & Zornia glochidiata Rchb. ex DC. & $\mathrm{Zgl}$ \\
\hline Capparaceae & Cleome viscosa $\mathrm{L}$ & Cvi \\
\hline \multirow{7}{*}{ Convolvulaceae } & Ipomoea asarifolia (Desr.) Roemer et J.A. Schultes & Ias \\
\hline & $\begin{array}{l}\text { Ipomoea coptica (L.) Roth ex Roemer et J.A. } \\
\text { Schultes }\end{array}$ & Ico \\
\hline & Ipomoea kotschiana L. & Iko \\
\hline & Ipomoea pes-tigridis L. & Ipe \\
\hline & Ipomoea sp L. & Isp \\
\hline & Jacquemontia tamnifolia (L.) Griseb. & Jta \\
\hline & Merremia tridentata (L.) Hallier f. & Mtr \\
\hline \multirow{5}{*}{ Cucurbitaceae } & Cucumus melo L. var agrestis Naudin & Cme \\
\hline & Cucumus metuliferus E Mey. Ex Naud & $\mathrm{Cmt}$ \\
\hline & Momordica balsamina $\mathrm{L}$. & Mba \\
\hline & Momordica charantia $\mathrm{L}$. & Mch \\
\hline & Mukia maderapatana (L.) M.Roem. & Mma \\
\hline Cyperaceae & Abildgaardia hispidula (Vahl) Lye & Ahi \\
\hline Euphorbiaceae & Chrozophora senegalensis (Lam.) A.Juss. ex Spreng. & Chs \\
\hline
\end{tabular}




\begin{tabular}{|c|c|c|}
\hline \multirow[t]{5}{*}{ Malvaceae } & Hibiscus physaloides Guill. \& Perr. & Hph \\
\hline & Hibiscus sabdarifa $\mathrm{L}$. & Hsa \\
\hline & Hibiscus sp L. & Hsp \\
\hline & Sida alba $\mathrm{L}$. & Sal \\
\hline & Waltheria indica $\mathrm{L}$. & Win \\
\hline \multirow[t]{2}{*}{ Pedaliaceae } & Ceratotheca sesamoides Endl. & Cse \\
\hline & Sesamum indicum $\mathrm{L}$. & $\operatorname{Sin}$ \\
\hline \multirow{13}{*}{ Poaceae } & Aristida adscensionis Linnaeus & Aad \\
\hline & Aristida stipoides Lam. & Ast \\
\hline & Brachiaria ramosa (Linn.) Stapf & Bra \\
\hline & Cenchrus biflorus Roxb. & Cbi \\
\hline & Chloris barbata $\mathrm{Sw}$. & $\mathrm{Cba}$ \\
\hline & Dactyloctenium aegyptium (L.) Willd & Dae \\
\hline & Digitaria horizontalis Willd. & Dho \\
\hline & Enteropogon prieurii (Kunth) Clayton & Epr \\
\hline & Eragrostis ciliaris (L.) var. ciliaris $\mathrm{R} . \mathrm{Br}$ & Epi \\
\hline & Eragrostis pilosa (L.) Beauv. & Etr \\
\hline & Eragrostis tremula Steud. & Eci \\
\hline & Panicum laetum Kunth & Pla \\
\hline & Schoenefeldia gracilis Kunth & Sgr \\
\hline Polygonaceae & Antigonum leptopus Hook. \& Arn. & Ale \\
\hline \multirow{4}{*}{ Rubiaceae } & Mitracarpus villosus (Sw.) DC. & Mvi \\
\hline & Spermacoce radiata (DC.) Hiern & Sra \\
\hline & Spermacoce rulliae DC. & Sru \\
\hline & Spermacoce stachydea DC. & Sst \\
\hline Blanophoraceae & Striga hermonthica (Del.) Benth & She \\
\hline Vitaceae & Cissus rufescens Guill. \& Perr. & $\mathrm{Cru}$ \\
\hline
\end{tabular}

Tableau 2: Valeur propre et pourcentage de variance des premiers axes de l'AFC appliquée aux relevés/espèces herbacées du milieu.

\begin{tabular}{lcccc}
\hline Axes & F1 & F2 & F3 & F4 \\
\hline Valeur propre & 0,515 & 0,297 & 0,273 & 0,238 \\
\% variance & 13,074 & 7,552 & 6,939 & 6,052 \\
\% cumulé & 13,074 & 20,625 & 27,564 & 33,616 \\
\hline
\end{tabular}


Tableau 3 : Paramètres physicochimiques des sols des différents groupements.

\begin{tabular}{lc|c|c|c}
\hline & \multicolumn{4}{c}{ Groupements végétaux } \\
\cline { 2 - 5 } Paramètres physicochimiques & $\begin{array}{c}\text { G à Aag } \\
\text { et Bra }\end{array}$ & $\begin{array}{c}\text { G à Tpu } \\
\text { et à Win }\end{array}$ & G à Zgl & $\begin{array}{c}\text { G à Aad et } \\
\text { Sgr }\end{array}$ \\
\hline Coefficient d'uniformité (CU) & 2,04 & 1,92 & 1,89 & 2,06 \\
Rapport C/N & 1,42 & 1,63 & 0,79 & 2,14 \\
Somme des bases échangeables ou S (méq/100g) & 1,63 & 1,47 & 1,35 & 1,98 \\
Na+ échangeable (méq/l) & 0,025 & 0,026 & 0,065 & 0,041 \\
Ph eau (1/2.5) & 7,3 & 8,3 & 7,3 & 8,6 \\
$\mathrm{Mg} / \mathrm{K}$ & 7,5 & 5 & 2,5 & 16,25 \\
$\mathrm{Ca}+\mathrm{Mg} / \mathrm{K}$ & 39,75 & 48 & 32,75 & 48,5 \\
$\mathrm{Ca} / \mathrm{Mg}$ & 4,3 & 8,6 & 12,1 & 1,98 \\
Fer libre (mg/l) & 3,15 & 2,87 & 3,06 & 3,27 \\
\hline
\end{tabular}

[G à Aag et à Bra = groupement à Achyranthes argentea Lam et à Brachiaria ramosa (L.) Stapf; G à Aad et à Sgr = groupement à Aristida adscensionis L. et Schoenefeldia gracilis Kunth; G à Tpu et à Win = à Tephrosia purpurea (L.) Pers. et Waltheria indica $\mathrm{L}$; $\mathrm{G}$ à $\mathrm{Zgl}=$ groupement à Zornia glochidiata Rchb. ex DC.]

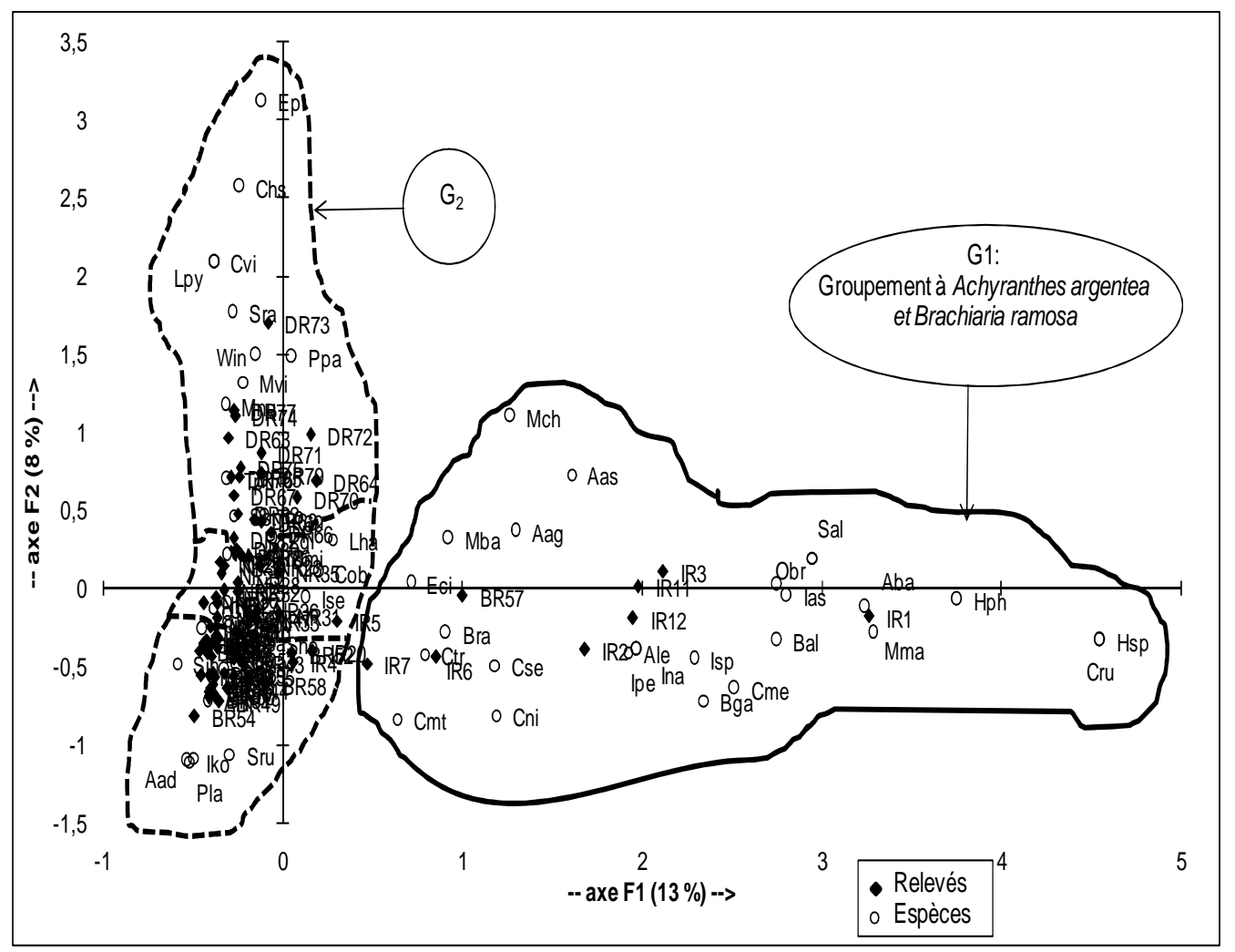

Figure 3 : Diagramme des 81 relevés et 62 espèces herbacées du milieu dans le plan des axes $F_{1}$ et $\mathrm{F}_{2}$. 


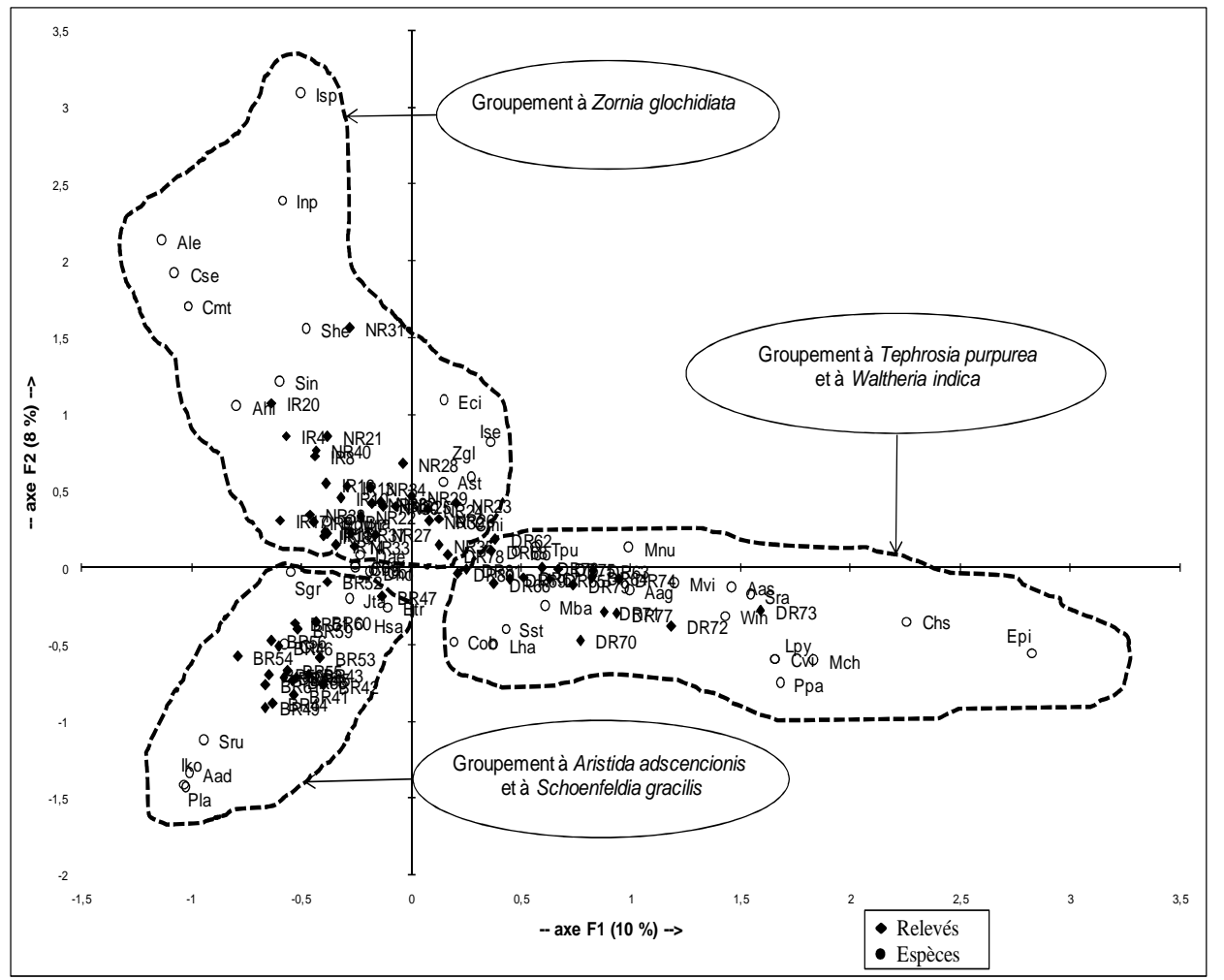

Figure 4 : Analyse partielle : distribution des espèces et relevés de Déali, Ndodj et Boulal dans le plan des axes 1-2.

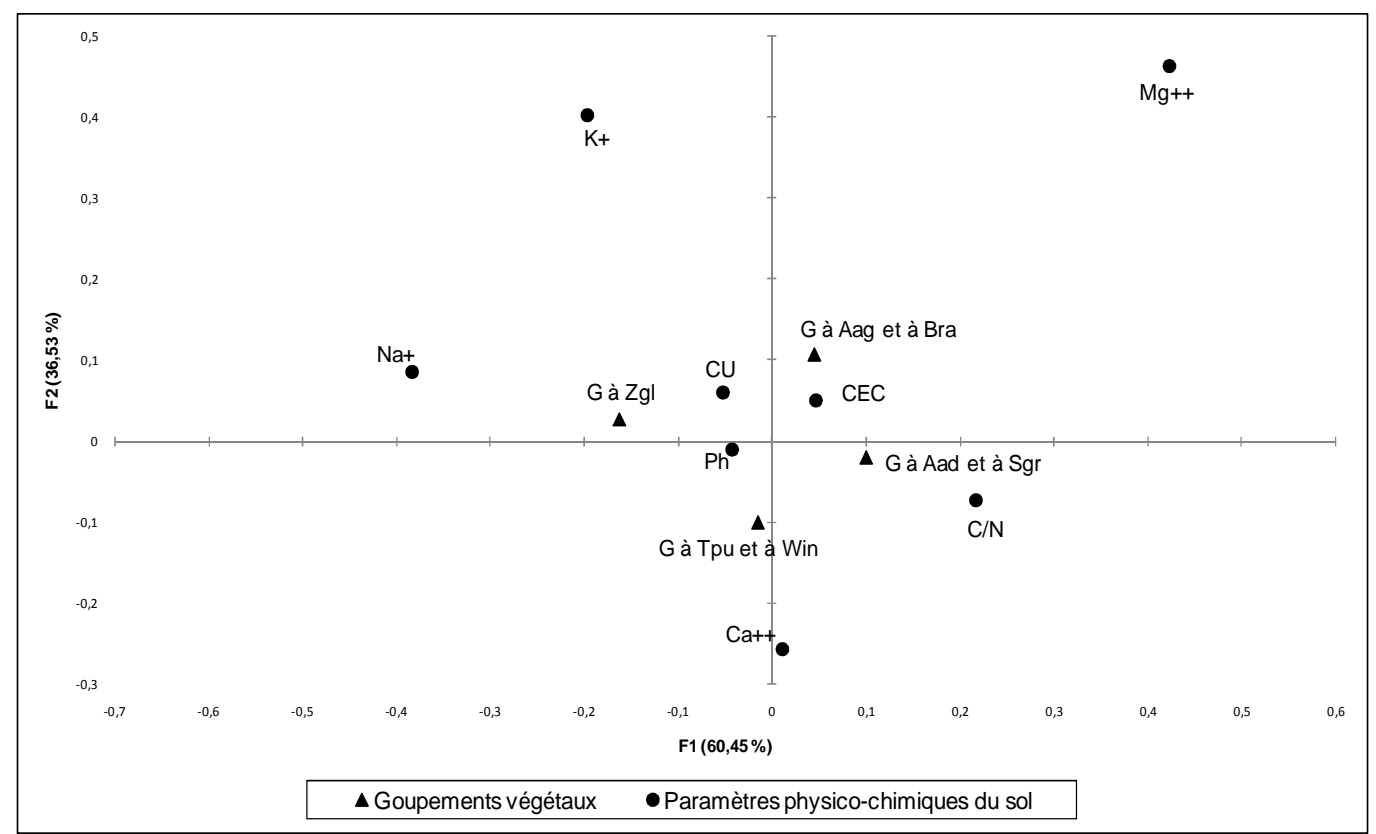

Figure 5 : - AFC : diagramme des groupements / paramètres physico-chimiques dans le plan des axes F1 (horizontal) et F2 (vertical). [G à Aag et à Bra = groupement à Achyranthes argentea Lam et à Brachiaria ramosa (L.) Stapf; G à Aad et à Sgr = groupement à Aristida adscensionis L. et Schoenefeldia gracilis Kunth; G à Tpu et à Win = à Tephrosia purpurea (L.) Pers. et Waltheria indica $\mathrm{L} . ; \mathrm{G}$ à Zgl = groupement à Zornia glochidiata Rchb. ex DC.] 


\section{DISCUSSION}

Les végétaux recensés dans la strate herbacée des plantations de Acacia senegal se répartissent en 62 espèces, 42 genres et 19 familles. Les résultats de la méthode des relevés phytosociologiques nous ont conduit à dégager quatre groupements végétaux distincts dans la strate herbacée. Ces résultats montrent qu'à une petite échelle, l'homogénéité de la végétation du Ferlo n'est qu'apparente. En effet, à une plus grande échelle, cette végétation se caractérise par une forte hétérogénéité (Akpo et al., 1995).

Les facteurs déterminants cette hétérogénéité relèvent d'une part, de facteurs abiotiques, essentiellement l'eau, la nature des sols et la topographie et d'autre part, de facteurs anthropiques liés surtout à l'action des troupeaux en raison de l'importance dans cette zone du pastoralisme.

Ainsi le groupement à Achyranthes argentea Lam et à Brachiaria ramosa (L.) Stapf est observé dans les bas-fonds (Isra, Boulal) inondés durant l'hivernage et formant des mares temporaires. Les sols de ces basfonds sont à granulométrie variée et étalée, neutres à légèrement alcalins. Ce groupement est beaucoup plus dense et très riche en Convolvulacées (Ipomoea pes-tigridis, Ipomoea asarifolia) occupant le centre des dépressions de la plantation Isra. Il serait analogue au groupement $\mathrm{II}_{\mathrm{c}}$ à Brachiaria ramosa décrit par Cornet et Poupon en 1978 et plus tard par Cornet en 1981 à Dahra sud.

Quant au groupement à Zornia glochidiata Rchb. ex DC., il occupe les zones de replat (Isra, Ndodj) sur un sol sableux fin légèrement alcalin. $\mathrm{Ce}$ groupement très appauvri, pourrait correspondre au facies de dégradation à Zornia glochidiata (groupement $\mathrm{I}_{\mathrm{c}}$ ) décrit toujours par les mêmes auteurs à Dahra sud.

Par contre, le groupement à Tephrosia purpurea (L.) Pers. et Waltheria indica L., pauvre en Graminées, colonise les bas de pente et les pentes (Déali) aux sols sableux fins fortement alcalins. Il correspondrait au groupement $\mathrm{I}_{\mathrm{b}}$ à Tephrosia purpurea que Cornet (1981) a décrit à Dahra nord.

Le groupement à Aristida adscensionis L. et Schoenefeldia gracilis Kunth occupe les dunes de Boulal dont les sols sableux moyens à fins sont fortement alcalins. Ce groupement est composé de plantes espacées recouvrant assez faiblement le sol. L'abondance ou la rareté de Schoenefeldia dans ce groupement dépend de la pluviosité de l'année. Il correspondrait au groupement $\mathrm{II}_{\mathrm{b}}$ à Schoenefeldia gracilis observé par Cornet à Dahra nord.

La végétation herbacée à Dahra est déséquilibrée dans sa répartition: elle est riche, diversifiée et forme un tapis continue dans les sites qui bénéficient d'une protection intégrale comme Isra; pauvre et discontinue dans les sites les moins protégés comme Déali. Elle est floristiquement pauvre à proximité des zones de cuvette mais avec un recouvrement très important par opposition à des endroits plus éloignés des points d'eau (dunes et replats) caractérisés par une grande richesse floristique mais à production faible. Dans les zones qui bénéficient d'une protection intégrale et les dépressions où la densité des ligneux est plus importante et les cimes des arbres plus ou moins jointives, nous avons noté une amélioration des conditions de développement de la strate herbacée avec une économie de l'eau, une atténuation des variations des paramètres climatiques, un enrichissement de la diversité herbacée comme certains auteurs (Akpo, 1993 ; Grouzis et Akpo, 1998) l'ont souligner dans d'autres endroits du Ferlo.

Le facteur hydrique est étroitement lié à la topographie et à la nature des sols. La répartition de l'eau est à l'origine de la différenciation des communautés spécifiques.

Aux alentours immédiats des zones de dépression, une diminution de la porosité des sols notamment en profondeur est notée. L'encroûtement superficiel des sols, contrairement aux observations de certains auteurs dans d'autres pays, ne peut pas être attribué dans le Ferlo sableux qu'aux effets du pâturage et du piétinement. Ce phénomène est 
rencontré dans les périmètres mis en défens durant plusieurs années et qui sont moins fréquentés. Il doit principalement être attribué à des facteurs liés à la texture et à la structure. Les sols sont en général neutres dans les profils des plantations Isra, Ndodj et dans les bas-fonds de la plantation Déali avec quelques horizons légèrement alcalins dans les zones de replat de Déali et dans la plantation Boulal. Ces sols pour la plupart neutres sont caractéristiques des sols ferrugineux tropicaux lessivés ou non sur les dunes et lessivés sur les plateaux anciens et les buttes résiduels décrits par la CSE en 2002. Ces sols sont marqués par la dominance de la fraction sableuse sur la majorité des horizons.

Tous les sols que nous avons caractérisé ont un taux en azote total inférieur à $0,37 \%$. Le rapport $\mathrm{C} / \mathrm{N}$ est très bas. $\mathrm{Au}$ vu de ces résultats et surtout ceux concernant la matière organique on pourrait en déduire qu'il y a un appauvrissement continu des sols en matière organique. Ceci peut être dû à plusieurs facteurs dont la disparition progressive de la végétation. Cet important rôle de la plante sur la fertilité des sols a été déjà énoncé par Akpo et Grouzis en 1996. Ces auteurs montrent que le taux de matière organique est de 1,5 à 5 fois plus important sous ombrage que dans une zone découverte. Duchaufour (2001) précise que c'est l'apport annuel de litières lors de la chute des feuilles qui renouvelle périodiquement le stock de matière organique existant en surface. Cet auteur indique que lorsque le sol forestier est couvert d'herbes (comme c'est le cas dans les bas-fonds), cette végétation herbacée intervient ainsi à hauteur de 40 à $50 \%$ de la litière sous la forme de matière organique riche en azote. La fertilité des sols, dans ces plantations, est dans l'ensemble médiocre. L'environnement de la région se dégrade en permanence $\mathrm{du}$ fait des facteurs d'ordre climatique, anthropique et animal.

L'effet de la pression anthropique sur les pâturages est surtout noté à Déali à proximité des zones de cuvettes formant des mares temporaires plus fréquentées par le bétail. La strate herbacée subit de profondes modifications dues à l'action des troupeaux comme l'ont montré les travaux de Valenza (1984) et Sy (2003). Dans les zones de replat et dunaires, la présence du bétail a une incidence sur la composition chimique du sol, favorisant l'implantation des espèces comme Schoenefeldia gracilis, Cenchrus biflorus, Dactyloctenium aegyptium et Zornia glochidiata qui profitent de l'apport d'azote. Par ailleurs, les espèces les plus sensibles aux piétinements telles que les grandes Andropogonées sont défavorisées et ont tendance à disparaître de la zone. La production primaire est plus élevée aux abords des bas-fonds fréquentés par le bétail du fait de la fertilité croissante des sols (Valenza, 1984) par rapport aux pâturages les plus éloignés. Cependant le surpâturage entraîne la disparition rapide de la strate herbacée soumettant le sol à une érosion éolienne et hydrique.

Dans les plantations d'Acacia senegal de la zone de Dahra, la végétation herbacée apparaît comme un ensemble de communautés complexes et très variables. Leur répartition dépend des caractéristiques des précipitations qui associées aux facteurs topographiques et édaphologiques amplifient l'hétérogénéité du milieu. La structure de la strate herbacée est aussi tributaire de la pression de pâture, c'est ainsi que dans les plantations qui bénéficient d'une protection intégrale le fourrage est présent en grande quantité et disponible jusqu'à des périodes très avancées de la saison sèche contrairement aux zones moins protégées où la dégradation de la végétation est beaucoup plus importante offrant des sols nus et pauvres en éléments minéraux.

Il importe de suivre les investigations afin d'établir la variabilité temporelle de cette végétation herbacée et d'évaluer ses interactions sur la structure et la production des peuplements d'Acacia senegal et sur le couvert ligneux en général. 


\section{REFERENCES}

Akpo LE. 1993. Influence du Couvert Ligneux sur la Structure et le Fonctionnement de la Strate Herbacée en Milieu Sahélien. Les Déterminants Ecologiques. Orstom éd : Paris, TDM ; $174 \mathrm{p}$.

Akpo LE, Gaston A, Grouzis M. 1995. Structure spécifique d'une végétation sahélienne. Cas de Wiidu Thiengoli (Ferlo, Sénégal). Bull. Mus. natl. Hist. nat., 17: 39-52.

Akpo LE, Grouzis M. 1996. Influence du couvert ligneux sur la régénération de quelques espèces ligneuses sahéliennes (Nord Sénégal, Afrique occidentale). Webbia, 19: 247-263.

Grouzis M, Akpo LE. 1998. Dynamique des interactions arbre/herbe en milieu sahélien. Influence de l'arbre sur la structure et le fonctionnement de la strate herbacée. In L'Acacia au Sénégal, Campa C, Grignon C, Guèye M, Hamon S (éds). Orstom édit: Paris.

Barral H, Bénéfice E, Boudet G. 1983. Systèmes de Production d'Elevage au Sénégal dans la Région du Ferlo. ACC GRIZA (LAT), Min. Rech. et Ind., GERDAT-ORSTOM: $172 \mathrm{p}$.

Bille JC. 1977. Etude de la Production Primaire Nette d'un Ecosystème Sahélien. Trav. et Doc. de l'ORSTOM ; 81 p.

Berhaut J. (1971-1979). Flore Illustrée du Sénégal. (tome I, 626 p.), (tome II, 695 p.), (tome III, 634 p.), (tome IV, 625 p.), (tome V, 658 p.), (tome VI, 636 p.). Gouvernement du Sénégal, Ministère du Développement rural, Direction des Eaux et Forêts, Dakar.

Braun-Blanquet J. 1928. Pflanzensoziologie. Grundzüge der Vegetationskunde. 1. Aufl. In Biologische Studienbücher 7, Schoenichen W (ed.). Springer Verlag: Berlin.

Carrière M. 1989. Les communautés végétales sahéliennes en Mauritanie (Région de Kaedi); analyse de la reconstitution annuelle du couvert herbacé. Thèse de doctorat en Sciences, Université de ParisSud, Centre d'ORSAY, 237 p.

Chevallier P, Claude J, Pouyaud B, Bernard A. 1985. Pluies et Crues au Sahel : Hydrologie de la Mare d'Oursi (Burkina Faso 1976-1981). Trav. et Doc. de I'ORSTOM: Paris; $251 \mathrm{p}$.

Cisse A. 1986. Dynamique de la Strate Herbacée des Pâturages de la Zone Sud Sahélienne. PPS, CABO ; 211 p.

Cornet A, Poupon H. 1978. Description des facteurs du milieu et de la végétation dans cinq parcelles situées le long d'un gradient climatique en zone sahélienne du Sénégal. Bull. IFAN, 39(2): 241-302.

Cornet A. 1981. Le bilan hydrique et son rôle dans la production de la strate herbacée de quelques phytocénoses sahéliennes au Sénégal. Thèse de Docteur-Ingénieur, Université des Sciences et Techniques du Languedoc, Montpellier, $354 \mathrm{p}$.

CSE (Centre de Suivi Ecologique). 2002. Synthèse des études diagnostiques des sites de l'observatoire du Ferlo. Projet ROSELT/OSS. Rap. Ministère de la jeunesse, de l'environnement et de l'hygiène publique. $10 \mathrm{p}$.

Dervin C. 1990. Comment Interpréter les Résultats d'une Analyse Factorielle des Correspondances? Mathématiques Informatique - Biométrie. I.N.R.A. I.N.A.P.G: Paris; 75 p.

Duchaufour P. 2001. Introduction à la science du sol, végétation, environnement. In Abrégé de Pédologie (6éme edn). 331 p.

Fournier A. 1991. Phénologie, Croissance et Productions Végétales dans quelques Savanes d'Afrique de l'Ouest. Variation selon un Gradient Climatique. Orstom édn : Paris ; 312 p.

Grouzis M, Legrand E, Pale F. 1986. Germination des semences des régions semi-arides du sahel. Actes du Colloque "Les Végétaux en Milieu Aride", ACCT Edn: TUNISIE-JERBA.

Grouzis M. 1988. Structure, Productivité et Dynamique des Systèmes Ecologiques 
Sahéliens (Mare d'Oursi, Burkina Faso). ORSTOM Edn: PARIS; 336 p.

Guinochet M. 1967. L'écologie végétale: quelques remarques sur ses fondements et ses objectifs. Mises à Jour Scient., 1: 387402.

Guinochet M. 1973. Phytosociologie. Masson: Paris; 227 p.

Hiernaux P. 1984. Distribution des pluies et production herbacée au sahel: une méthode empirique pour caractériser la distribution des précipitations journalières et ses effets sur la production herbacée. Premiers résultats acquis dans le sahel malien, ILCA, Bamako, 46 p.

Lacoste A, Salanon R. 1969. Eléments de Biogéographie et d'Ecologie. Nathan. Coll. Fac. Géographie: Paris; 189.

Lebrun JP, Stork AL. Enumération des Plantes à Fleurs d'Afrique Tropicale. Conservatoire du Jardin Botanique de Genève, I, II, III, IV, 1991, 1992, 1995 et $1997: 249,257,341$ et 712 .

Menaut JC. 1983. The vegetation of african savannas. In "Ecosystems of the World",Tropical Savannas (n॰13). Bourlière (Ed). Elsevier Scient. Publi. : Oxford ; 109-149.

Michel P. 1969. Les bassins des fleuves Sénégal et Gambie. Etude géomorphologique. Doctorat ès Sc., Strasbourg, $1167 \mathrm{p}$.

Penning de Vries FWT, Djiteye MA. 1982. La Productivité des Pâturages Sahéliens. Une Etude des Sols, Végétations et de l'Exploitation de cette Ressource Naturelle. CABO: Wageningen; 525 p.

PEPAM 2007. Plan local d'hydraulique et d'assainissement-plha. Communauté Rurale de Labgar. [en ligne]. Disponible à
http://www.pepam.gouv.sn/PLHA/PLHA Labgar.pdf [cité le 12 mars 2010].

Poissonet J, César JC. 1972. Structure spécifique de la strate herbacée dans la savane à palmier rônier de Lamto (Côte d'Ivoire). Ann. Univ. Abidjan, 5: 577-601.

Poupon H. 1980. Structure et Dynamique de la Strate Ligneuse d'une Steppe Sahélienne au Nord du Sénégal. Trav. et Doc. de I'ORSTOM ; 317 p.

Raynal J. 1964. Etude botanique des pâturages du Centre de Recherches Zootechniques de Dahra-Djoloff (Sénégal). Rapp. ORSTOM, Dakar, 99 p.

Sy O. 2003. Dynamique des ressources en eau et évolution de la mobilité pastorale en Zone sylvopastorale (ZSP), Thèse de doctorat de $3^{\text {ème }}$ cycle, Dakar, UCAD-ISE, $217 \mathrm{p}$.

Toutain B, Bortolli L, Dulieu D, Forgiarini G, Menaut JC, Piot J. 1983. Espèces Ligneuses et Herbacées dans les Ecosystèmes Sahéliens Pâturés de HauteVolta. ACC GRIZA (LAT), GERDAT ; 124.

Trochain J. 1940. Contribution à l'étude de la végétation du Sénégal. Mém. IFAN Dakar, 433 p.

Valenza J, Diallo K. 1972. Etudes des pâtures du Nord Sénégal. IEMVT. Etude Agrostologie, 34. Maisons-Alfort ; 331. Une carte au 1/200 000 en trois feuilles.

Valenza J. 1984. Surveillance continues des pâturages naturels sahéliens sénégalais Résultats de 10 années d'observation. Dakar. AGROSTO, 2(44): 81. 